\title{
Effect of Intestinal Microbiota on Brain Activity: ANN Analysis of Functional Magnetic Resonance Imaging
}

\author{
Bouharati Imene ${ }^{1,2}$, Bouharati Khaoula ${ }^{3}$, Boubendir Nacereddine ${ }^{4}$, Laouamri Slimane ${ }^{2}$ \\ ${ }^{1}$ Laboratory of intelligent systems, UFAS Ferhat Abbas Setif University, Algeria, \\ ${ }^{2}$ Faculty of Medicine, UFAS Ferhat Abbas Setif University, Algeria, \\ ${ }^{3}$ Faculty of Medicine, Constantine University, Algeria \\ ${ }^{4}$ Faculty of Medicine, Algiers University, Algeria \\ E-mail: imene.bouharati@univ-setif.dz
}

\begin{abstract}
Modern biotope genomics has been able to determine the characteristics of each individual. The intestinal microbiota has a direct effect on the brain and behavioral activity of the individual. Any change in it modifies the mechanisms of emotional signaling, behavioral and visceral nociceptive reflexes. The resulting signaling mechanisms of the gut microbiota and probiotics including variations in microbiota production influence brain activity. Studies on rodents with reported vagal signaling in response to intestinal and probiotic pathogens. Diet, genetic factors, environmental factors may play a role in these alterations. The result is that is proven by functional brain imaging. The resulting effect of this interaction can be recorded in specific areas in brain using functional magnetic resonance imaging. Depending on different biotopes in different people, levels of stress or relaxation are recorded. The analysis of these variations, allows having an idea on the effect carried by each alteration in biotope and drugs administered to soothe stress for example. However, these effects are far from accurate. Human physiology is much more complex to draw hasty conclusions. If the effect is much more pronounced in women is that they are more emotional, sex is a factor to consider. In this study, we propose an intelligent system for analyzing these variables. The analysis of the MRI image is very complex due to it is a question of defining the exact contours of the activated brain zones. Given the complexity of the system, an artificial neural network analysis with deep learning is proposed. The constructed system is supervised learning. Input variables are (Biotope classification, genetic factor, diet factor, sex) and an output variable that expresses the degree of effect on activation of brain areas in relation to the centers of emotions recorded in functional fMRI. The established algorithm randomly introduces values to the input factors of the system to read predict the emotional effect on the individual.
\end{abstract}

Keywords:Biotope, intestinal microbiota, cerebral activity, fMRI, ANN, Deep learning

\section{Introduction}

The chemistry of the central nervous system is modified by the intestinal microbiota. This plays a vital role in bidirectional interactions between the gut and the nervous system. The regulation of neuronal activators by the biotope acts on the endocrine system. The result is a direct influence on the emotional aspect such as anxiety, stress and even memory functions ${ }^{[1]}$. What is noted is that these disorders are directly related to chronic bowel disorders even in those with inflammations in the gastrointestinal tract ${ }^{[2]}$. Experiments on the animal model demonstrate that there is a behavioral change in subjects with acute and chronic gastrointestinal inflammation. But the exact mechanism of action is not yet well defined ${ }^{[3,4,5,6]}$.

This gut-brain interaction has been observed for a long time. It is only in recent years that she has regained interest by focusing on diet that has a direct effect on the microbiotabrain axis. Recent studies focus on vague nerve, immune system, microbial metabolites, tryptophan metabolism, and intestinal hormone signaling ${ }^{[7]}$.

All these interactions produce activations of specific brain areas. In functional brain magnetic resonance imaging, it is possible to locate these areas. However, it is very difficult to say precisely on the correspondence between these recorded areas and the effect of the microbiota. Activation of these areas may be the result of other intrinsic factors. Also, the sensitivity of the magnetic field makes it can be influenced even by the slightest movement of the patient including his breathing. Also, because the principle of magnetic resonance imaging is based on measurements of blood oxygenation and contrast (BOLD signal). This technique measures neuronal activity, but it accurately measures blood volume and 
oxygenation. This variation in oxygenation of the blood volume indirectly reflects the neuronal activity ${ }^{[8]}$. Given this complexity of the system, a technique of artificial intelligence including artificial neural networks with supervised learning is proposed in this analysis. The constructed system consists of the inputs that characterize the factors involved in this effect and an output that represents the registered fMRI image. The input and output values are introduced with all combinations from real cases. The correspondence between the two input-output spaces is established in this learning phase. Once the transfer function is established with the minimum of error, it will be possible to predict the effect of each factor on the emotional state of the patient.

\section{Role of the Microbiome}

The microbiom plays a role more and more known in recent years in determining behavior ${ }^{[9,10,11,12]}$. This role is already involved in the development of the brain. During its development, the brain is influenced by the microbiome ${ }^{[13]}$. The experiments carried out on mice during the faecal transplantation of one presenting a certain behavior, this one is translated on the recipient ${ }^{[14]}$. which proves the direct effect and the role played by the microbiome. Even in humans, experiments show that the microbiota of the mucosa of subjects with cirrhosis, exhibit cognitive disorders ${ }^{[15]}$. Other studies suggest that the neurochemical compound produced by the biotope may be at the origin of the behavioral definition in animals and humans ${ }^{[16]}$. The effect of bacterial metabolites on the central nervous system may be due to the absorption of bacterial products and its circulation in the blood system. Also, interactions of these compounds with the nerves or endocrine cells at the level of the intestine can explain this interaction. Toll-like receptors directly influence lipopolycaccharides and have a direct effect on the central nervous system ${ }^{[17,18]}$. By this, the permeability of the intestines is a determining factor in this process ${ }^{[19]}$. On the other hand, the brain influences the microbiota in an indirect way and that by the action on the intestinal transit and the secretions. This action occurs through molecular release prompting signals in the lumen of the intestine ${ }^{[20]}$. This is accompanied by variations in biological parameters including action on mediators of systemic stress ${ }^{[21]}$.

\section{Microbiota and Brain Imaging}

The action of the central nervous system on the functions of the body defines the emotional motor system. This one refers to different parallel outputs. The brain areas concerned are the sympathetic system, the parasympathetic branches, the autonomic system, the hypothalamus, the pituitary-adrenal axis, as well as the endogenous pathways responsible for the modulation of pain, comfort and emotional state in general [22].

To illustrate these effects, it is important to locate these areas in cerebral imaging which allows to measure emotions. This has been proven experimentally by ingestion of probiotics and its effect on brain function in women. What is recorded is that it produces a direct action on areas involved in the recognition of emotions ${ }^{[23]}$. Studies are conducted in brain imaging by combining the microbial ecology of the intestine taking into account the large-scale neural network ${ }^{[24,25]}$. This work will have to specify how the microbiota influences the brain and determine the mechanism by which changes related to psychiatric symptoms occur ${ }^{[26]}$.

\section{System Characterization}

The information on the mechanisms governing the micobiotebrain relation is very poor despite the irrefutable proof of this link. But this field offers great opportunities for research and future prospects ${ }^{[27]}$. Clinical studies on phenotyped patients throughout their lives make it possible to follow the evolution of the composition of the microbiota under the effect of probiotics or antibiotics. The results will be of great value for future research ${ }^{[28]}$.

In this study, our interest lies in the localization of the incriminated brain zones in the emotional changes under the effect of the intestinal microbiota. However, the images recorded in functional MRI are far from precise and refer to the effect of the microbiota. Other factors influence the emotional state. Also, in magnetic resonance imaging, sensitivity is greatly increased. Several parasitic factors disturb the magnetic field and induce erroneous images.

The cerebral areas that are thought to be involved in the emotional state and thus related to the psychiatric symptoms, namely the sympathetic system, the parasympathetic branches, the autonomic system, the hypothalamus, the pituitary-adrenal axis, as well as the endogenous pathways, are considered as output variables of the proposed system. Factors causing such activation zones are considered as input variables of the system. These factors are probiotic phenotype, diet, sex, age and genetic factor. The factors considered in this study are exhaustive and the system allows its extension to other variables not supported in this study.

\section{Artificial neural networks and deep learning}

In imitation of the biological neural network, the mathematicians have tried to reproduce this diagram in algorithmic. Artificial neural networks are highly connected networks making the correspondence between two spaces (input-output). These networks have proved their validity in different scientific, technological and medical fields ${ }^{[29]}$. From experimental data, artificial neural networks have the dynamics and ability to read data and solve complex systems [30].

The proposed neural network is multi-layered. If this technique is a deep learning often applied in machine learning in the field of artificial intelligence, its application in the medical field requires a lot of data. Deep learning is more 
effective when computational resources are wide ${ }^{[31]}$. In our case, the complexity of the system and data relating to the location of the brain zones activated by the various factors and especially the gut microbiota makes the application of such a system proves to be adequate.

In our case, it is to build the network (Figure 1) and make the correspondence between the input and output variables mentioned above.

\section{Input variables}

Each variable is numerically coded

Probiotic phenotype: is coded in three phynotypic categories $(1 ; 2 ; 3)$

Diet: is coded in three diet types $(1 ; 2 ; 3)$

Sex: is coded in two gender, 1 for male and 2 for female

Genetic: is coded in three interval's effect $(1 ; 2 ; 3)$

Age: the age is represented by its numeric values in years which range from 0 to 100 years

\section{Output variables:}

Activated zones recorded in functional MRI The activated area returns numerical values that represent each part.

1 for sympathetic system,

2 for parasympathetic branches,

3 for autonomic system,

4 for hypothalamus,

5 for pituitary-adrenal axis,

6 for endogenous pathways

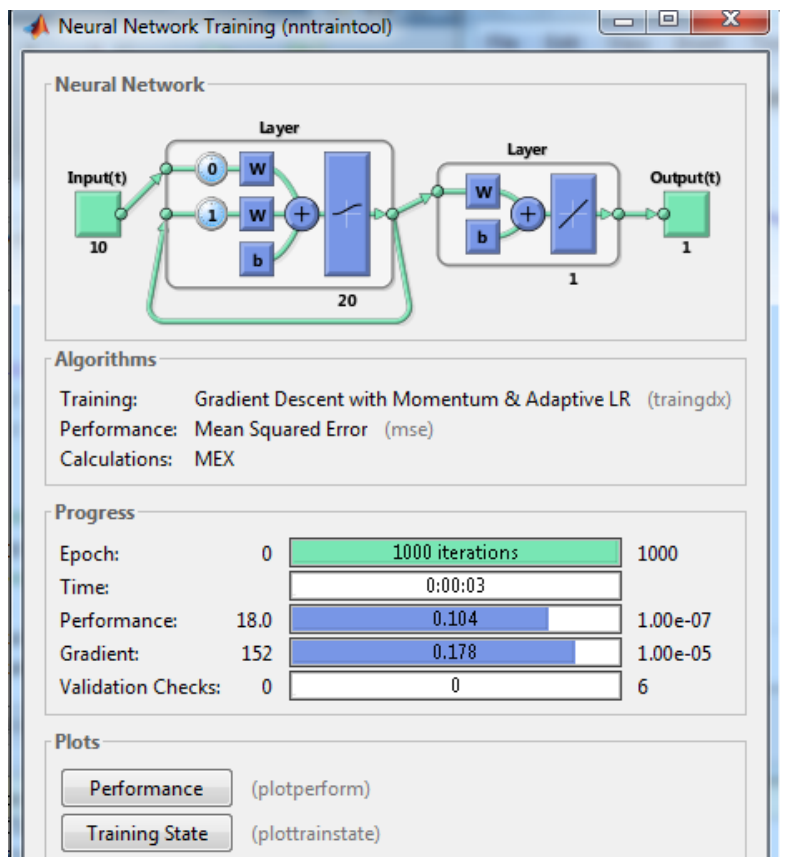

Figure 1. Block diagram of the system

\section{Learning the neural network}

The input values are set according to the result at the output, i.e. the registered brain area. The system establishes a transfer function that links the input to the output. At each inputoutput match line, the system adjusts the function. During this learning phase, the system adjusts the function by acting on the weights of each connection. These weights are simply mathematical coefficients. So, the advantage is that it is not necessary to create a new function with each new given, but just the network operates by coefficient variations up to the optimum of the function with the minimum of errors. By entering the maximum number of variables to encompass all possible combinations, it becomes possible to enter random variables to automatically read the cerebral zone that will be activated.

\section{Result and discussion}

The encoded data are presented in Excel file format. Learning is done by reading input-output data. These data are recorded from real cases. Learning is done from line-by-line reading. The transfer function is created and optimized. The remaining intermediate lines are left for the test phase. 1000 loop iterations are programmed to achieve the optimal fit. At 1000 iterations, the performance of the system using the least squares method is 10-7 with a gradient of 10-5 (Figure 1). The optimum of the function reaches 1000 epocs with an error of 0.1038 (Figure 2).

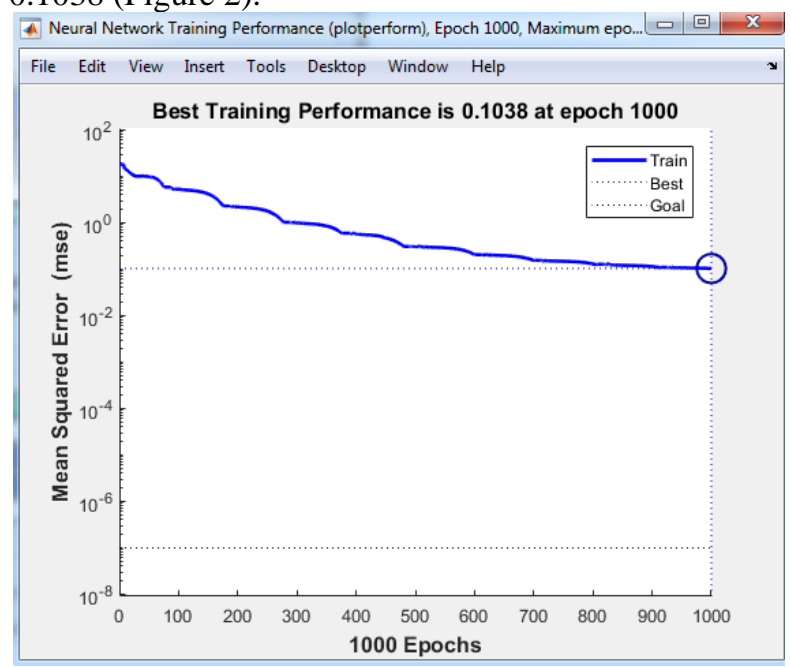

Figure 2. Training system

\section{Conclusion}

The biotope-brain interaction is a proven. The effect of each on the other is crossed. The effect of the gut microbiota on the brain is manifested by activation of one or more areas in the brain. These areas are mainly related to the emotional center. This is reflected in return times by a physical action of the brain. The identification of these areas with precision is very difficult due to several factors they can intervene there. In view of the complexity of the system related to these multitudes of factor interactions and also to the sensitivity of functional magnetic resonance imaging, the proposed system 
allows these complexities to be addressed. In this study, after collecting the majority of the factors as input variables from the system and matching fMRI images as output variables, this makes it possible to assign the result to the input and vice versa. The proposed artificial neural network system processes all possible combinations recorded. The learning of the multilayer network (input layer, hidden layer and output layer) in deep learning makes it possible to have an optimal input output function. From there, it becomes possible to introduce variables at the input to automatically and instantly predict the result at the output by referring to the function created in learning. Thus, the brain areas that will activate will be planned. As these areas are directly related to the emotional state of the patient, it then becomes possible to predict the effect of food or antibiotic for example on the state of stress or moral comfort of the person. The proposed system allows for extension to other factors that are not considered in this study. Thus, the accuracy will be even more increased.

\section{References}

[1]. Bajaj J.S., Hylemon P.B., Ridlon J.M., Heuman D.M., Daita K., White M.B., Monteith P., Noble N.A., Sikaroodi M., Gillevet P.M.. 2012. Colonic mucosal microbiome differs from stool microbiome in cirrhosis and hepatic encephalopathy and is linked to cognition and inflammation. Am J Physiol Gastrointest Liver Physiol 303:G675-685.

[2]. Bercik P., Denou E., Collins J., Jackson W., Lu J., Jury J., Deng Y., Blennerhassett P., Macri J., McCoy K.D., Verdu E.F., Collins S.M.. 2011a. The intestinal microbiota affect central levels of brainderived neurotropic factor and behavior in mice. Gastroenterology 141:599-609.

[3]. Bercik P., Verdú E.F., Foster J.A et al. 2009. Role of gut-brain axis in persistent abnormal feeding behavior in mice following eradication of Helicobacter pylori infection. Am J Physiol Regul Integr Comp Physiol. 296:R587-94. [PubMed: 19129375]

[4]. Bercik P., Verdu E.F., Foster J.A. et al. 2010. Chronic gastrointestinal inflammation induces anxiety-like behavior and alters central nervous system biochemistry in mice. Gastroenterology. 139:2102-12. [PubMed: 20600016]

[5]. Bouharati I., Bouharati K., Bouharati S. and HamdiChérif M. 2017. Functional magnetic resonance imaging: fuzzy inference analysis. Radiology and Diagnostic Imaging.. Volume 1(2): 1-4. doi: 10.15761/RDI.1000111

[6]. Bravo J.A., Forsythe P., Chew M.V., Escaravage E., Savignac H.M., Dinan T.G., Bienenstock J., Cryan J.F. 2011. Ingestion of Lactobacillus strain regulates emotional behavior and central GABA receptor expression in a mouse via the vagus nerve. Proc Natl Acad Sci USA 108:16050-16055.

[7]. Collins S.M., Kassam Z., Bercik P. 2013. The adoptive transfer of behavioral phenotype via the intestinal microbiota: experimental evidence and clinical implications. Curr Opin Microbiol 16:240245.

[8]. Collins S.M., Kassam Z., Bercik P. 2013. The adoptive transfer of behavioral phenotype via the intestinal microbiota: experimental evidence and clinical implications. Curr Opin Microbiol 16:240245.

[9]. Cryan J.F., Dinan T.G. 2012. Mind-altering microorganisms: the impact of the gut microbiota on brain and Behavior. Nat Rev Neurosci 13:701-712.

[10]. Daniele R., Charence W., Fani D., Melissa B., Javier A.P., Benny L.O., and Guang-Zhong Y. 2017. Deep Learning for Health Informatics. IEEE Journal of Biomedical and Health Informatics, Vol. 21, No. 1, January. 4-20.

[11]. Douglas-Escobar M., Elliott E., Neu J. 2013. Effect of intestinal microbial ecology on the developing brain. JAMA Pediatr 167:374-379.

[12]. Foster A.J., Linda R., Cryan F.J. Stress \& the gutbrain axis: Regulation by the microbiome. Neurobiology of Stress 7 124e136. 2017

[13]. Foster J.A., McVey Neufeld K.A. 2013. Gut-brain axis: how the microbiome influences anxiety and depression. Trends Neurosci 36:305-312.

[14]. Goehler L.E., Gaykema R.P., Opitz N. et al. 2005. Activation in vagal afferents and central autonomic pathways: early responses to intestinal infection with Campylobacter jejuni. Brain Behav Immun. 19:33444. [PubMed: 15944073]

[15]. Irimia A., Van Horn J.D. 2013. The structural, connectomic and network covariance of the human brain. Neuroimage 66:489-499.

[16]. Jane A.F., Mark L., Emeran M., and John F.C. 2016. Gut Microbiota and Brain Function: An Evolving Field in Neuroscience. International Journal of europsychopharmacology, 19(5): 1-7.

[17]. Katherine L. and L. Sandrine T. 2015. Gut Microbiota: A Modulator of Brain Plasticity and Cognitive Function in Ageing. Healthcare, 3, 898916; doi:10.3390/healthcare3040898

[18]. Khaoula B., Mustapha B., Saddek B., Mokhtar H.C. 2017. Leishmaniasis transmission vectors analysis using artificial neural networks. Averooes European Medical Journal. Volume 5, Number 1.

[19]. Khenchouche A., Bouharati K., Bouharati S., Mahnane A., Hamdi-Cherif M. 2017. Post Mortem Interval: Necrobiome Analysis Using Artificial Neural Networks. Computational Biology and Bioinformatics. 5(6): 90-96. 
[20]. Kim K.A., Gu W., Lee I.A., Joh E.H., Kim D.H. 2012. High fat diet-induced gut microbiota exacerbates inflammation and obesity in mice via the TLR4 signaling pathway. PLoS One.7:e47713.

[21]. Lyte M., Varcoe J.J., Bailey M.T. 1998. Anxiogenic effect of subclinical bacterial infection in mice in the absence of overt immune activation. Physiol Behav. 65:63-8. [PubMed: 9811366]

[22]. Lyte M.M. 2013. Microbial endocrinology in the microbiome-gutbrain axis: how bacterial production and utilization of neurochemicals influence behavior. PLOS Pathog 9:e1003726.

[23]. Marilia C., Annunziata S., Masellib A.M., Carola S. 2015. The gut-brain axis: interactions between enteric microbiota, central and enteric nervous systems. Annals of Gastroenterology. 28, 203-209.

[24]. Mayer E.A. 2000. The neurobiology of stress and gastrointestinal disease. Gut.; 47:861-869. [PubMed: 11076888]

[25]. O'Mahony L. et al. 2005. Lactobacillus and bifidobacterium in irritable bowel syndrome: symptom responses and relationship to cytokine profiles. Gastroenterology.; 128:541-551. [PubMed: 15765388

[26]. Rhee H.S., Charalabos P., Mayer E.A. 2009. Principles and clinical implications of the brain-gutenteric. microbiota axis. Nat Rev Gastroenterol Hepatol. 6(5): 1-19.

[27]. Saulnier D.M., Riehle K., Mistretta T.A., Diaz M.A., Mandal D., Raza S., Weidler E.M., Qin X., Coarfa C., Milosavljevic A., Petrosino J.F. , Highlander S., Gibbs R., Lynch S.V., Shulman R.J., Versalovic J. 2011. Gastrointestinal microbiome signatures of pediatric patients with irritable bowel syndrome. Gastroenterology 141:1782-1791.

[28]. Shi H., Kokoeva M.V., Inouye K., Tzameli I., Yin H., Flier J.S. 2006. TLR4 links innate immunity and fatty acid-induced insulin resistance. J Clin Invest. 116:3015-3025.

[29]. Stephen M.C., Michael S. and Premysl B. 2012. The interplay between the intestinal microbiota and the brain. Nature Rev. Microbiol. 2012 Nov;10(11):73542.

[30]. Tillisch K., Labus J., Kilpatrick L., Jiang Z., Stains J., Ebrat B., Guyonnet D., Legrain-Raspaud S., Trotin B., Naliboff B., Mayer E.A. 2013. Consumption of fermented milk product with probiotic modulates brain activity. Gastroenterology 144:1394-1401.

[31]. Tillisch K., Labus J., Kilpatrick L., Jiang Z., Stains J., Ebrat B., Guyonnet D., Legrain-Raspaud S., Trotin B., Naliboff B., Mayer E.A. 2013. Consumption of fermented milk product with probiotic modulates brain activity. Gastroenterology 144:1394-1401.

[32]. Whitehead W.E., Palsson O., Jones K.R.. 2002 Systematic review of the comorbidity of irritable bowel syndrome with other disorders: what are the causes and implications? Gastroenterology. 122:1140-56. [PubMed: 11910364]

[33]. Yarandi S.S., Peterson D.A., Treisman G.J., Moran T.H., and Pasricha J.P. 2016. Modulatory Effects of Gut Microbiota on the Central Nervous System: How Gut Could Play a Role in Neuropsychiatric Health and Diseases. J Neurogastroenterol Motil. 22:201-212. 